\title{
The conservation pattern of short linear motifs is highly correlated with the function of interacting protein domains Siyuan Ren ${ }^{1,3}$, Guang Yang1, Youyu $\mathrm{He}^{2}$, Yiguo Wang ${ }^{1}$, Yixue $\mathrm{Li}^{2}$ and Zhengjun Chen*1
}

Address: ${ }^{1}$ State Key Laboratory of Molecular Biology, Institute of Biochemistry and Cell Biology, Chinese Academy of Sciences, Shanghai, PR China, ${ }^{2}$ Bioinformatics Center, Shanghai Institutes for Biological Sciences, Chinese Academy of Sciences, Shanghai, PR China and ${ }^{3}$ Center for Information Science and Technology, Temple University, Philadelphia, PA 19122, USA

Email: Siyuan Ren - rsysjtu@hotmail.com; Guang Yang - gyang@sibs.ac.cn; Youyu He - yyhe@sibs.ac.cn; Yiguo Wang -wangyg@sibs.ac.cn; Yixue Li - yxli@sibs.ac.cn; Zhengjun Chen* - zjchen@sibs.ac.cn

* Corresponding author

Published: I October 2008

BMC Genomics 2008, 9:452

doi:10.1186/|47|-2164-9-452
Received: 4 March 2008

Accepted: I October 2008

(c) 2008 Ren et al; licensee BioMed Central Ltd.

This is an Open Access article distributed under the terms of the Creative Commons Attribution License (http://creativecommons.org/licenses/by/2.0), which permits unrestricted use, distribution, and reproduction in any medium, provided the original work is properly cited.

\begin{abstract}
Background: Many well-represented domains recognize primary sequences usually less than 10 amino acids in length, called Short Linear Motifs (SLiMs). Accurate prediction of SLiMs has been difficult because they are short (often $<10$ amino acids) and highly degenerate. In this study, we combined scoring matrixes derived from peptide library and conservation analysis to identify protein classes enriched of functional SLiMs recognized by SH2, SH3, PDZ and S/T kinase domains.

Results: Our combined approach revealed that SLiMs are highly conserved in proteins from functional classes that are known to interact with a specific domain, but that they are not conserved in most other protein groups. We found that SLiMs recognized by $\mathrm{SH} 2$ domains were highly conserved in receptor kinases/phosphatases, adaptor molecules, and tyrosine kinases/ phosphatases, that SLiMs recognized by SH3 domains were highly conserved in cytoskeletal and cytoskeletal-associated proteins, that SLiMs recognized by PDZ domains were highly conserved in membrane proteins such as channels and receptors, and that SLiMs recognized by S/T kinase domains were highly conserved in adaptor molecules, $S / T$ kinases/phosphatases, and proteins involved in transcription or cell cycle control. We studied Tyr-SLiMs recognized by $\mathrm{SH} 2$ domains in more detail, and found that SH2-recognized Tyr-SLiMs on the cytoplasmic side of membrane proteins are more highly conserved than those on the extra-cellular side. Also, we found that $\mathrm{SH} 2-$ recognized Tyr-SLiMs that are associated with $\mathrm{SH} 3$ motifs and a tyrosine kinase phosphorylation motif are more highly conserved.
\end{abstract}

Conclusion: The interactome of protein domains is reflected by the evolutionary conservation of SLiMs recognized by these domains. Combining scoring matrixes derived from peptide libraries and conservation analysis, we would be able to find those protein groups that are more likely to interact with specific domains. 


\section{Background}

Selective protein-protein interactions are important for cellular functions and are often mediated by protein domains that recognize specific primary sequences within target proteins called Short Linear Motifs (SLiMs). Accurate prediction of SLiMs has been difficult because they are short (often $<10$ amino acids) and highly degenerate. A major advance in SLiM identification came with a peptide library-based technique that can map the sequence motif recognized by an $\mathrm{SH} 2$ domain without prior knowledge of in vivo interaction sites [1]. Similar peptide library experiments have been performed to map the motifs recognized by other domains. Motifs discovered through polypeptide library screening have shown high levels of agreement with reported domain interaction sites $[1,2]$. This became the basis for Scansite [3,4], a bioinformatics program developed to predict SLiMs in query proteins that are recognized by specific protein domains. Other bioinformatic approaches, like those available in Minimotif-Miner [5], QuasiMotifFinder [6], MCS [7] and a treebased scoring [8] applied evolutionary conservation as well as other sequence filters to assess the functional relevance of a hit.

Both peptide library screening and evolutionary conservation proved to be useful in prediction motifs, we hypothesized that combining chemical enrichment scoring matrixes derived from peptide libraries and conservation analysis would discriminate between classes of proteins that have functional SLiMs and those that do not. To address this issue, we conducted a global statistical analysis on the conservation of SLiMs recognized by $\mathrm{SH} 2, \mathrm{SH} 3$, PDZ and S/T kinase domains (Invariant features in SLiMs recognized by each domain were shown in Table 1) in different functional classes of proteins. For each domain we studied, our analysis revealed that domain-recognized SLiMs are highly conserved in specific functional classes of proteins that are known to frequently interact with that domain, but they are not conserved in most other protein groups. For example, we found that SLiMs that interact with SH2 domains are conserved in receptor kinases/ phosphatases, adaptor molecules, and tyrosine kinases/ phosphatases. Our analysis also confirmed that most SH2-mediated signaling occurs in the cytoplasm, and suggests that SLiMs that are recognized by tyrosine kinases

Table I: Invariant features in SLiMs recognized by $\mathrm{SH} 2, \mathrm{SH} 3$, PDZ and S/T Kinase domains

\begin{tabular}{lll}
\hline Domain & SLiM & length \\
\hline SH2 & YXXX & 4 \\
SH3 Type I & XXXPXXP & 7 \\
SH3 Type 2 & XPXXPXX & 7 \\
PDZ & XXXXX-COOH & 5 \\
S/T Kinase & XXXXS/TXXXX & 9 \\
\hline
\end{tabular}

and are in proteins that contain multiple $\mathrm{SH} 3$ binding motifs are more likely to interact with SH2 domains.

\section{Results}

\section{Relative Conservation $\left(C_{R}\right)$ of SLiMs}

Relative conservation of SLiMs was measured to assess their functional importance. The central hypothesis was that SLiMs should be subject to two kinds of evolutionary selection. The first is background selection, which is imposed upon the entire length of the protein sequence, and is due to factors such as the overall stability, structure, and function of the protein. The second is SLiM-specific selection superimposed on the background, due to the special function mediated by the SLiM. Therefore, a wellconserved SLiM in an overall highly conserved protein does not guarantee independent importance. For example, although the two putative SH2 binding Tyr-SLiMs in Histone H3.1 were conserved among sequences from all selected species (Figure 1A upper panel), their relative conservation was low because of the highly conserved background (see Figure 1A lower panel for a schematic illustration of the background and SLiM specific relative conservation and alignment of Histone H3.1 Y54). It is possible Tyr-SLiMs in Histone H3.1 are conserved because they have an integral function in protein structure or stability. Conversely, a SLiM with high relative conservation is an indication that the motif may play a unique physiological role. The five Tyr-SLiMs in the C-terminus of IL4R are well conserved, while the full-length protein is not (Figure 1B upper panel); thus, these SLIMs have a high level of relative conservation (see Figure 1B lower panel for a schematic illustration of the relative conservation and alignments of IL4R Y631 and Y821). In fact, four of these five conserved tyrosine motifs are reported to bind to $\mathrm{SH} 2$ domains [9]. The relative conservation method allows us to discriminate between SLiMs that have been conserved due to structural constraints of the protein from those that have been conserved to serve as functional motifs. We do not argue against the importance of conserved motifs in conserved proteins; however, we consider them less likely to function independently.

\section{Analysis of SH2 Domain-Mediated Signaling in I I Highly Studied Receptor Tyrosine Kinases (RTKs)}

To test the functional relevance of our SLiM conservation calculation, we analyzed reported SH2 binding sites in 11 highly-studied RTKs (with greater than 30 interaction partners, according to Hprd), including EGFR, IR, KIT, PDGFRB, IGF-IR, VEGFR2, ERBB2, FGFR1，HGFR，RET and TKR-A. We manually extracted interactions from the literature between one of these RTKs and one of the 21 $\mathrm{SH} 2$ domains we are studying here, which yielded a total of 76 interactions involving 56 unique Tyr-SLiMs (refer to Table S1 for detail). Using our SLiM conservation calculation, we found that reported SH2 binding sites have significantly higher $(\mathrm{p}<0.0001$, Mann-Whitney test $) \ln \left(\mathrm{C}_{\mathrm{R}}\right)$ 

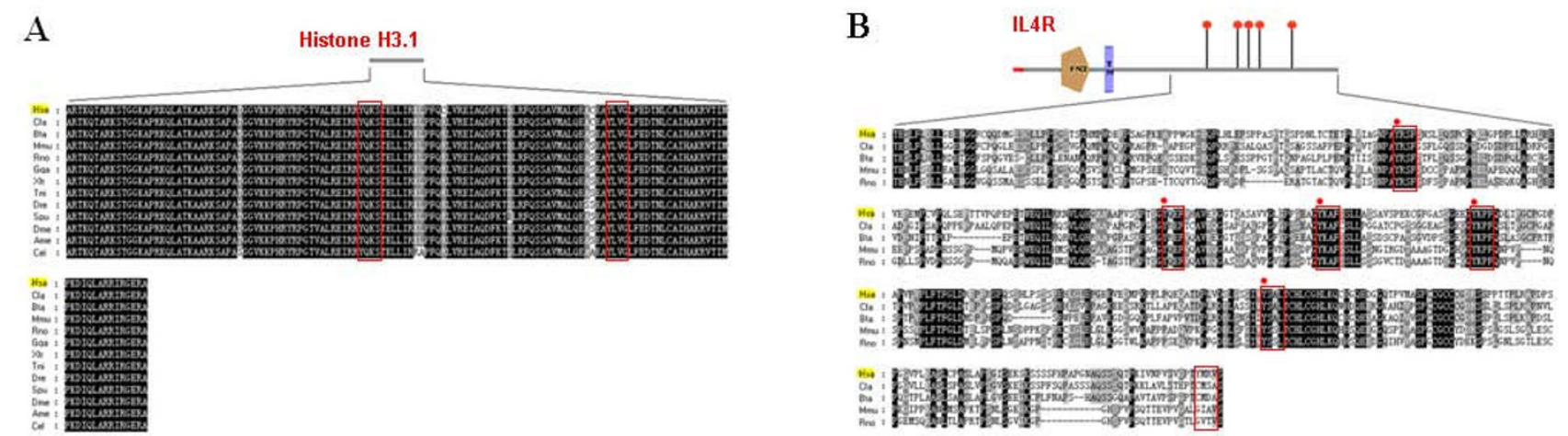
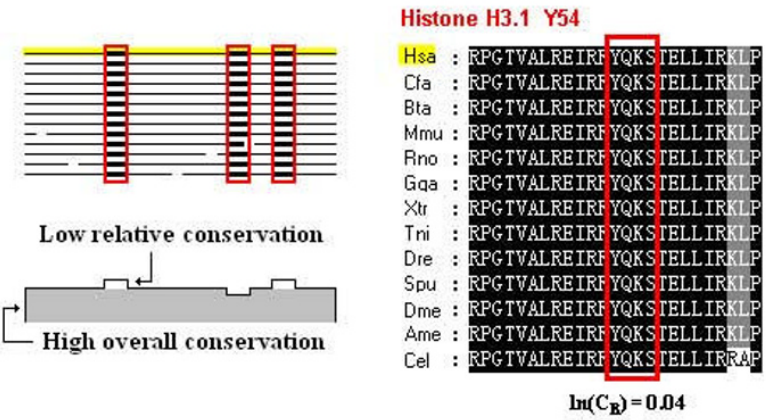

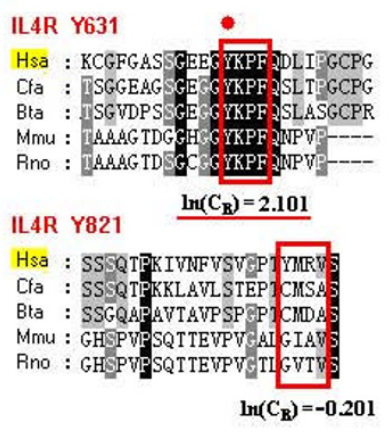

Figure I

Relative conservation of SLiMs. (A) Low relative conservation of a conserved SLiM in an overall highly conserved protein. Sequence alignment of Histone H3.I with potential SH2 binding Tyr-SLiMs in red boxes (upper panel). Schematic illustration and alignment around Y54 of Histone H3.I are shown below. (B) High relative conservation of a conserved SLiM in an overall less-conserved protein. C-terminal sequence alignment of IL4R with potential SH2 binding Tyr-SLiMs shown in red boxes (upper panel). Schematic illustration of relative conservation and alignment around Y63I and Y82I of IL4R are shown below.

scores (which measures the relative conservation of a motif) than those sites that do not bind to $\mathrm{SH} 2$ domains (Figure 2A), indicating the relative conservation score is an effective distinguishing factor of functional binding SLiMs.
In order to evaluate the specificity of motif prediction, we compared the SH2 selectivity values (which is calculated using enrichment values from peptide library screening) of SLiMs in proteins from reported binding groups to the $\mathrm{SH} 2$ selectivity values of SLiMs in proteins from groups
A

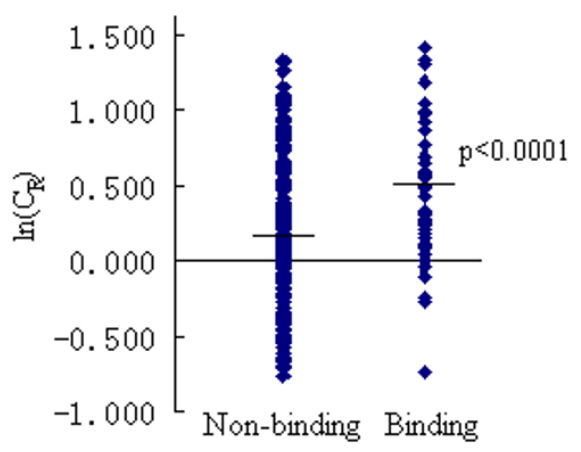

B

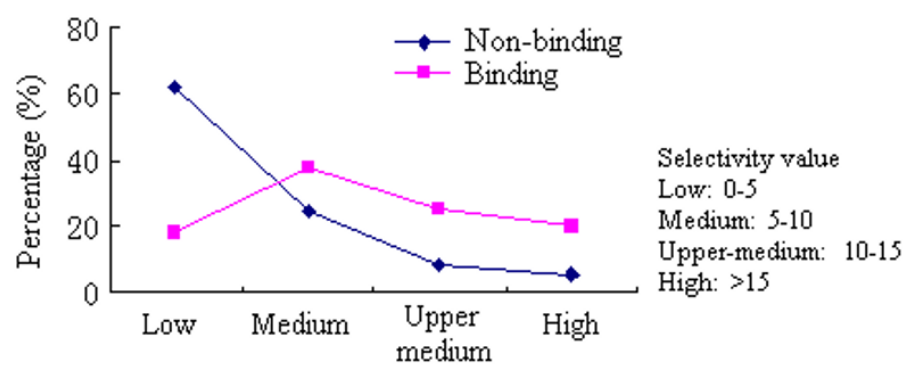

SH2 selectivity value

Figure 2

Comparison of conservation scores and $\mathbf{S H} 2$ selectivity values between $\mathrm{SH} 2$ binding and non-binding TyrSLiMs in I I highly studied RTKs. (A) SH2 binding Tyr-SLiMs are significantly more conserved than those that do not bind to $\mathrm{SH} 2$ domains ( $\mathrm{p}<0.000 \mathrm{I}$, Mann-Whitney test). (B) Percentage of SLiMs that have different $\mathrm{SH} 2$ selectivity values in binding and non-binding groups. 
that are not reported to bind. We found that less than $40 \%$ of non-binding SLiMs have a selectivity value > 5, whereas over $80 \%$ of binding SLiMs have a selectivity value greater than 5 . Higher selectivity values correspond to a higher specificity of interaction (Figure $2 \mathrm{~B}$ ). These results demonstrate that predicting domain binding to SLiMs based on motifs from peptide library experiments is effective.

\section{Global Conservation Analysis of SLiMs Recognized by SH2, SH3, PDZ and S/T Kinase Domains}

Using the PLC- $\gamma 1 \mathrm{~N}$-terminal SH2 domain as a model to study the relationship between conservation and function of SLiMs, we found that Tyr-SLiMs predicted to bind to the PLC- $\gamma 1$ N-terminal SH2 domain (selectivity value $\geq 5.0$ ) have significantly higher $\ln \left(\mathrm{C}_{\mathrm{R}}\right)$ scores, compared to TyrSLiMs in PLC- $\gamma 1$ binding proteins (Mann-Whitney test, $\mathrm{p}$ $=0.001$; Fig. 3A, left panel, III) and receptor kinase/phosphatase proteins ( $\mathrm{p}=0.002$; Fig. $3 \mathrm{~A}$, left panel, II) not predicted to bind to the $\mathrm{N}$-terminal $\mathrm{SH} 2$ domain (selectivity value $<5.0)$. No significant increase in $\ln \left(\mathrm{C}_{\mathrm{R}}\right)$ score was observed for $\mathrm{SH} 2$-recognized ( $\mathrm{SH} 2$ selectivity $\geq 5.0$ ) TyrSLiMs in cell cycle control proteins ( $\mathrm{p}>0.3$, Fig. 3A, left panel, I); importantly, cell cycle control proteins are rarely reported to bind to $\mathrm{SH} 2$ proteins.

Taking into consideration all binding partners, we found that for 20 of the 21 SH2 motifs, Tyr-SLiMs recognized by SH2 domains (selectivity value $\geq 5$ ) have a higher average $\ln \left(C_{R}\right)$ score than those not recognized by SH2 domains (selectivity value $<5$ ); 11 of these are statistically significant $(\mathrm{p}<0.05)$. In the receptor kinase and phosphatase group, 8 cases showed a significant increase in $\ln \left(C_{R}\right)$ score. However, no significant increase in $\ln \left(\mathrm{C}_{\mathrm{R}}\right)$ score was observed in the cell cycle control protein group (Figure $3 \mathrm{~A}$, right panel).

We then systematically examined the conservation of SLiMs recognized by $\mathrm{SH} 2, \mathrm{SH} 3, \mathrm{PDZ}$ and S/T Kinase domains (selectivity value $\geq 5$ ) in representative protein functional classes taken from the Hprd database (Figure $3 \mathrm{~B})$. Those functional groups that show significant increase of conservation highly correlated with those that frequently interact with respective domains (functional classes frequently reported to interact with each domain were listed in Table 2). We observed that SH2-recognized SLiMs (Figure 3B, first panel) are most highly conserved in receptor kinases/phosphatases, adaptor molecules, tyrosine kinases/phosphatases and structural proteins; conservation was occasionally found in cytokine/immune receptors, cell junction proteins and cytoskeletal-associated proteins. Most other functional protein classes had little conservation signal. This result correlated well with those protein functional groups frequently interact with $\mathrm{SH} 2$ proteins as listed in Table 2 . There are also some sporadic signals such as Nck in adhesion molecules and
GTPase, Vav in cytoskeletal proteins and SHC in DNA binding proteins, suggesting they may interact with proteins in those functional groups.

For SH3-recognized SLiMs (Figure 3B, second panel), conservation was strongest in cytoskeletal and cytoskeletalassociated proteins. calcium binding proteins, RNA binding proteins, tyr-kinases/phosphatases and guanine nucleotide exchange factors also had strong conservation signals. The conservation signal was almost absent in other functional classes. This is largely consistent with those frequently reported $\mathrm{SH} 3$ interacting protein groups (Table 2).

Consistent with biochemical evidences that PDZ domains frequently interact with membrane proteins, we found that PDZ domain-recognized SLiMs (Figure 3B, third panel) are specifically conserved in membrane proteins including channels, integral membrane proteins, cell surface receptors, $\mathrm{G}$ protein/G protein coupled receptors and membrane transport proteins. The frequent interacting partners of PDZ domain containing proteins are channels, adhesion molecules and cell surface receptors (Table 2). Our results suggest that those membrane proteins such as integral membrane proteins were probably less well studied but nevertheless play an important role in interaction with PDZ domain.

As shown in Figure 3B, fourth panel, the proteins containing SLiMs recognized by $\mathrm{S} / \mathrm{T}$ kinases in the basophilic group (basophilic S/T kinases in this study included AKT, PKA, PKC, SRPK2, Clk2, NIMA, PhK, CamK2, SLK and MAPKAPK2) seem to be involved in a wider variety of cellular functions than proteins with SLiMs recognized by SH2, SH3 and PDZ domains. S/T kinase domain-recognized SLiMs were conserved in proteins involved in signal transduction (adaptor proteins and Ser/Thr kinase/phosphatases), in cytoskeletal-associated proteins, in proteins related to transcription and cell-cycle control, and also in some membrane proteins. However, the proteins containing conserved SLiMs recognized by proline-dependent Ser/Thr kinases (including CDK2, CDC2 and CDK5) were more specifically involved in transcription and cell-cycle control, with almost no conservation signal from other functional categories. The conservation pattern of SLiMs recognized by S/T kinases is highly consistent with protein functional groups with high serine phosphorylation ratio (Table 2).

Remarkably, most functional classes of proteins with a significant conservation signal were highly specific for the signal within one group of domains, but not in other groups. For example, receptor kinase/phosphatase group show conservation signal only in SH2 domain group and transcription factors only in Ser/Thr kinase domain group 
A

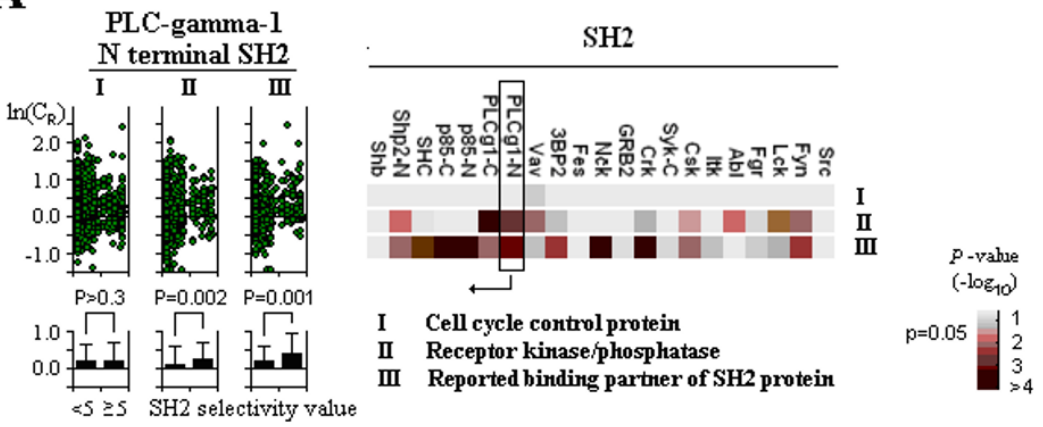

B
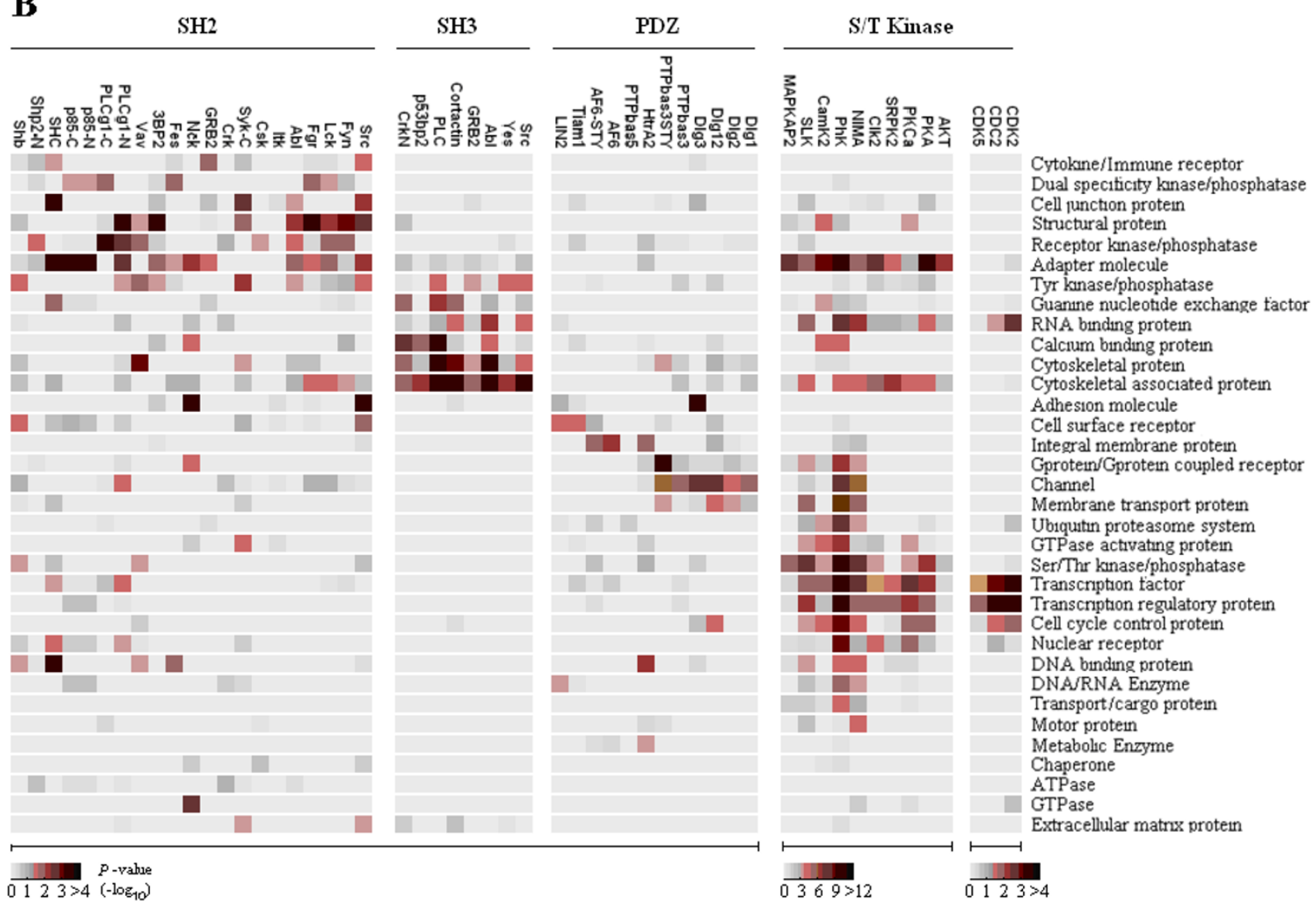

Figure 3

Conservation analysis of SLiMs recognized by SH2, SH3, PDZ and S/T kinase domains in different protein functional classes. (A) Conservation analysis of potential SH2 binding Tyr-SLiMs in cell cycle control proteins, receptor kinases/ phosphatases and reported SH2 binding partners. The PLC-gamma-I N terminal SH2 domain is shown as an example (left panel). The $\mathrm{x}$-axis represents the selectivity of the PLC-gamma-I N terminal SH2 domain, and the $y$-axis indicates the logarithm of $C_{R}$. The Mann-Whitney test was performed to calculate the significance of the increase of conservation between $\mathrm{SH} 2 \mathrm{non}$ selected (selectivity value $<5$ ) and selected (selectivity value $\geq 5$ ) Tyr-SLiMs. A color-coded map of $p$-values is shown on the right. (B) Conservation analysis of SLiMs recognized by SH2, SH3, PDZ and S/T kinase domains in different protein functional groups. Color-coded maps of $\mathrm{p}$-values are shown below. 
Table 2: Molecular functional classes frequently reported to interact with $\mathrm{SH} 2, \mathrm{SH} 3$ or PDZ domains, or to be phosphorylated by $\mathrm{S} / \mathrm{T}$ kinases

\begin{tabular}{llc}
\hline Domain & Molecular function & Binding ratio* \\
\hline SH2 & Receptor kinase/phosphatase & 0.53 \\
& Tyrosine kinase/phosphatase & 0.51 \\
& Cytokine/lmmune receptor & 0.36 \\
& Adapter molecule & 0.20 \\
& Cell surface receptor & 0.14 \\
$\mathrm{SH} 3$ & Tyrosinekinase/phosphatase & 0.32 \\
& Adapter molecule & 0.18 \\
& Guanine nucleotide exchange factor & 0.12 \\
& Cytoskeletal protein & 0.11 \\
& GTPase activating protein & 0.11 \\
PDZ & Channel & 0.214 \\
& Adhesion molecule & 0.075 \\
& Cell surface receptor & 0.052
\end{tabular}

\begin{tabular}{llc}
\hline Kinase & & Phospho ratio\# \\
\hline S/T Kinase & Serine/threonine kinase/phosphatase & 0.00442 \\
& Cell cycle control protein & 0.00397 \\
& RNA-binding protein & 0.00334 \\
& Transcription factor & 0.00320 \\
& Adapter molecule & 0.00296 \\
& Structural protein & 0.00259 \\
& Transcription regulatory protein & 0.00255 \\
\hline
\end{tabular}

* The binding ratio is calculated as the percentage of proteins that interact with proteins containing $\mathrm{SH} 2, \mathrm{SH} 3$, or PDZ domains. \# The phosphorylation ratio is calculated as the ratio of serine residues that are phosphorylated.

(Figure 3B) Nevertheless, a few protein functional classes exhibited a significant conservation signal in multiple groups of domains, such as adaptor molecules and cytoskeletal-associated protein groups; this corresponds to the fact that these proteins participate in multiple signaling pathways involving interactions with more than one domain.

In order to further examine the specificity of the conservation signal, we calculated the conservation profile of SLiMs in each protein functional class by calculating the difference in $\ln \left(\mathrm{C}_{\mathrm{R}}\right)$ score between SLiMs with high selectivity and those with low selectivity. We also compared functional classes that are frequent, occasional or rare interaction partners for each domain by setting thresholds for the percentage of proteins in the functional class that either interact with or become phosphorylated by proteins containing that domain (Refer to Additional File 1 for detail. Frequent interaction partners for each domain were listed in Table 2). As expected, the conservation signal was highest in functional classes of proteins that are frequently reported to interact with a specific domain, and the signal progressively decreased for functional protein classes that are reported to interact occasionally or rarely with binding partners (Figure 4). Conservation profiles calculated as the change in $\ln \left(C_{R}\right)$ score between SLiMs with upper-medium to medium selectivity values and SLiMs with low selectivity values showed similar trends, but were less significant (Figure S2, S3). In the above experiments, SLiMs for SH2 domains, PDZ domains or S/ $T$ Kinases with selectivity values of $<5,5-10,10-15$, and $>15$ were assigned to the categories of low, medium, upper medium and high selectivity, respectively; SLiMs with SH3 selectivity values of $<3,3-6,6-9$, and $>9$ were assigned to the categories of low, medium, upper medium and high selectivity, respectively.

\section{Conservation of SLiMs in Sub-Cellular Localization and in Multi-Domain Signaling}

Using SH2 domain-interacting SLiMs as a model, we applied our method of conservation analysis to study additional aspects of SLiM conservation. Specifically, we investigated the conservation of SLiMs in proteins that interact with two different protein domains in a signaling pathway, and we studied the relationship between conservation of SLiMs and sub-cellular localization.

Consistent with the observation that $\mathrm{SH} 2$-mediated signaling mainly occurs in the cytoplasm, we found a conservation signal for SH2-recognized SLiMs in cytoplasmic but not extra-cellular regions in both Type I and II membrane proteins (Figure 5A). (For Type I membrane proteins, the cytoplasmic side is C-terminal, while for Type II membrane proteins it is $\mathrm{N}$-terminal.) Since the majority of membrane proteins are Type I, we further classified this group by protein function. The conservation signal is strongest for SLiMs on the cytoplasmic side of receptor kinases/phosphatases, cell surface receptors, cytokine/ immune receptors and adhesion molecules, and weaker for SLiMs in channels and metabolic enzymes (Figure 5B). SH2-domain binding is dependent on tyrosine phosphorylation, which is catalyzed by Tyr kinases. Accordingly, SLiMs recognized by Tyr kinases should be more likely to interact with SH2 domains. We found that SH2-recognized SLiMs that were selected for based on the presence of a common tyrosine kinase motif (containing E/D up to four amino acids from the tyrosine on the N-terminal side) are more conserved than those without this selection (Figure 6A).

On the other hand, many tyrosine kinases (including the well-known Src family kinases) and adaptor molecules have both SH2 and SH3 domains, and it has been suggested that proteins containing multiple $\mathrm{SH} 3$ binding sites are more likely to be tyrosine phosphorylated and bind to $\mathrm{SH} 2$ domains as supported by biochemical studies $[10,11]$. Consistent with this reasoning, SH2-recognized Tyr-SLiMs in signal transduction proteins that have more than ten PXXP SH3 binding motifs are significantly more conserved than SLiMs without this selection (Figure 

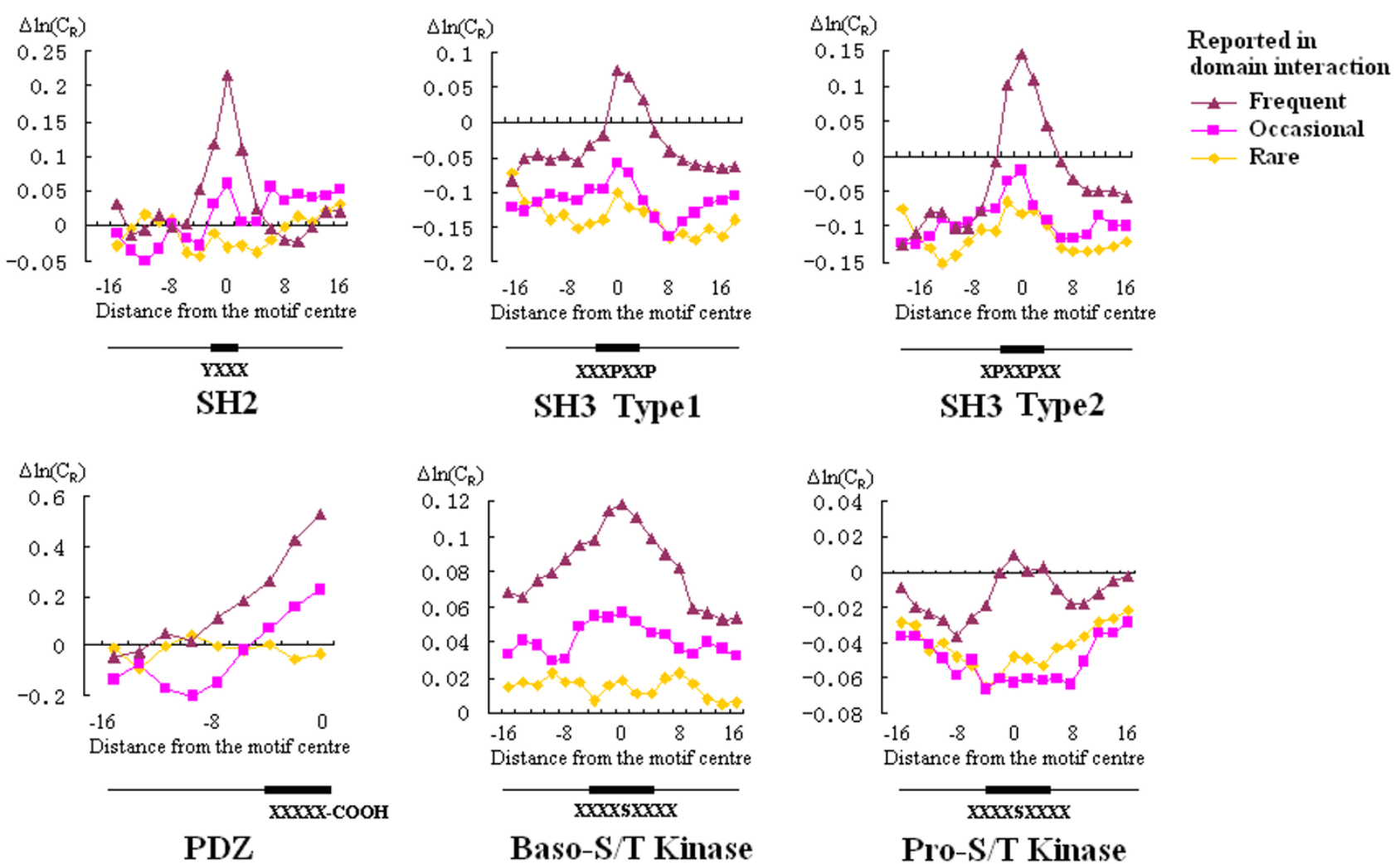

\section{Figure 4}

Averaged conservation profiles of functional classes that are frequent, occasional or rare interaction partners of specific domains. The plots show the change in $\ln \left(C_{R}\right)$ between sequences containing SLiMs with high selectivity values and those containing SLiMs with low selectivity values for specific domains. The approximate SLiM regions are indicated with black boxes. Domain-recognized SLiMs are most conserved in protein functional classes that frequently interact with a specific domain.

6A). However, this trend is not observed in SLiMs in functional classes other than signal transduction protein class (Figure 6A), which agrees well with the fact that most SH2-binding proteins are signal transduction proteins.

We further divided signal transduction groups into subgroups according to sub-cellular localization. Under selections for both the kinase motif and SH3 binding motifs, a high level of SLiM conservation was most manifest in signal transduction proteins localized to the cytoplasm or plasma membrane, but conversation of SLiMs was weaker for those proteins localized to the nucleus (Figure 6A). This is consistent with biochemical evidence that tyrosine phosphorylation occurs mainly in the cytoplasm and plasma membrane (the ratios of proteins that bind to SH2-containing proteins in the cytoplasm, plasma membrane and nucleus are $16.1 \%, 11.4 \%$ and $4.7 \%$ respectively, according to Hprd). Conservation profiles for different functional classes of proteins with or without
SH3 and Tyr-Kinase domain selection are shown in Figure $6 \mathrm{~B}$.

These findings support the hypothesis that tyrosine kinases and SH3 domains are frequently coupled to $\mathrm{SH} 2$ domain signaling. The coupling between a tyrosine kinase and $\mathrm{SH} 2$ domains is expected, since an $\mathrm{SH} 2$ domain can only bind to a Tyr-SLiM after the tyrosine residue has been phosphorylated by a Tyr-kinase. However, the coupling between SH2 and SH3 domains might be less direct. Either a sequential model or a cooperative model, depending on whether the target tyrosine residue is phosphorylated before the interaction, may be used to explain the coupling between SH2 and SH3 domains (Figure 6C). In the sequential model, PXXP motifs recruit SH3 domain containing Tyr-kinases, which in turn phosphorylate the tyrosine residues in the target protein. The pYXXX motif can then recruit an $\mathrm{SH} 2$ domain (Figure 6C, upper panel). In the cooperative model, the $\mathrm{SH} 2$ and $\mathrm{SH} 3$ domains in a single kinase or adaptor molecule bind to the pYXXX 
A

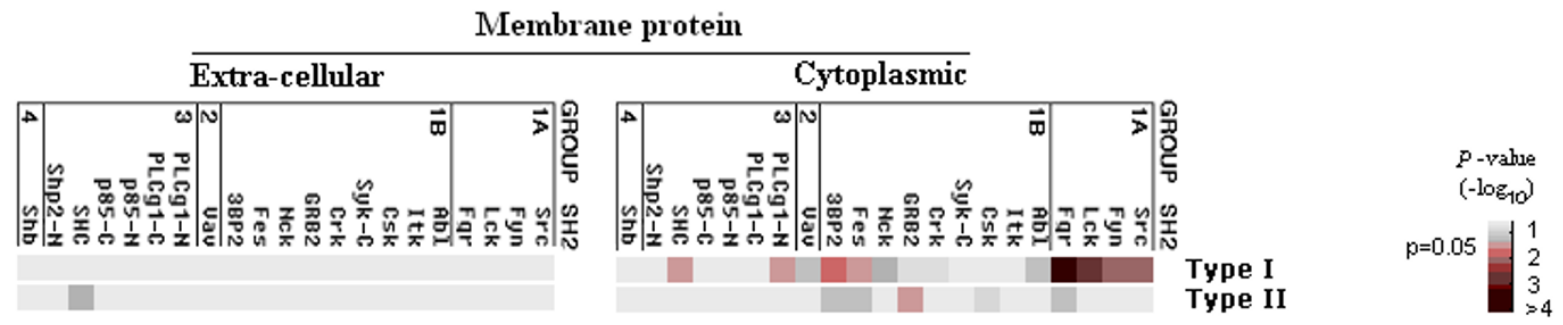

Type I membrane protein
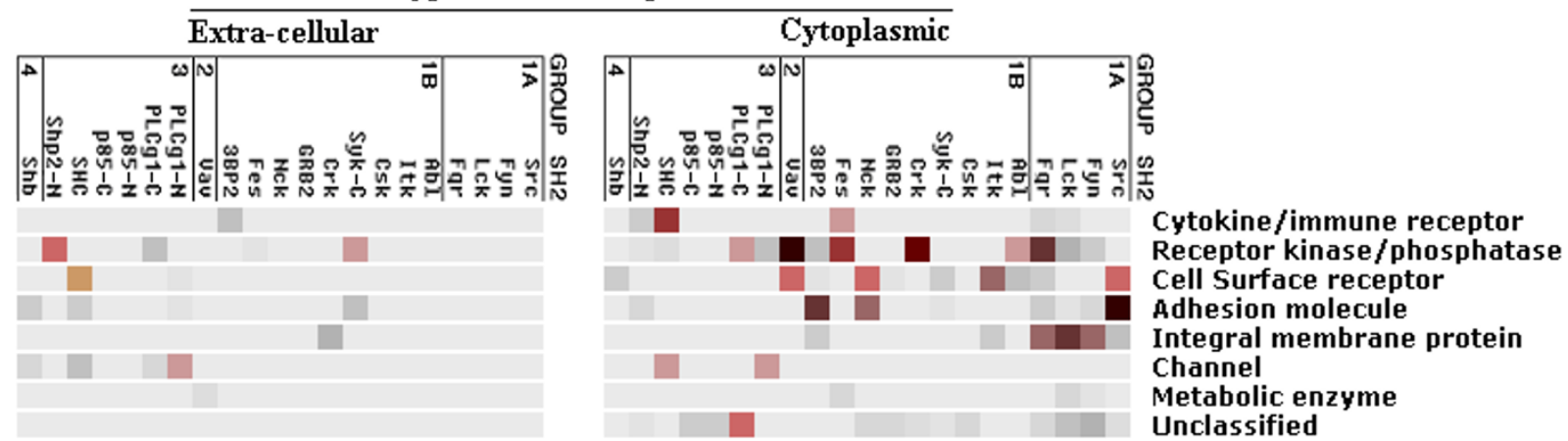

B

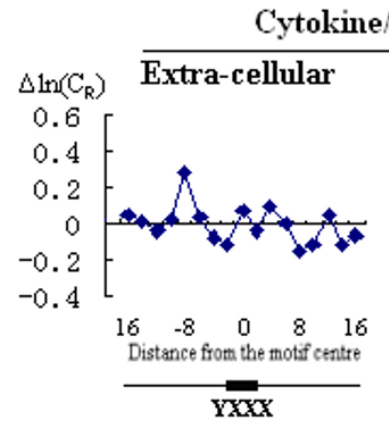

Cytokine/Immune receptor

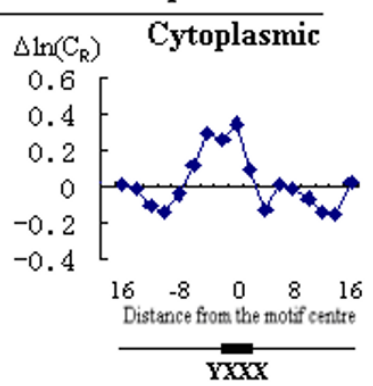

Cell sufface receptor
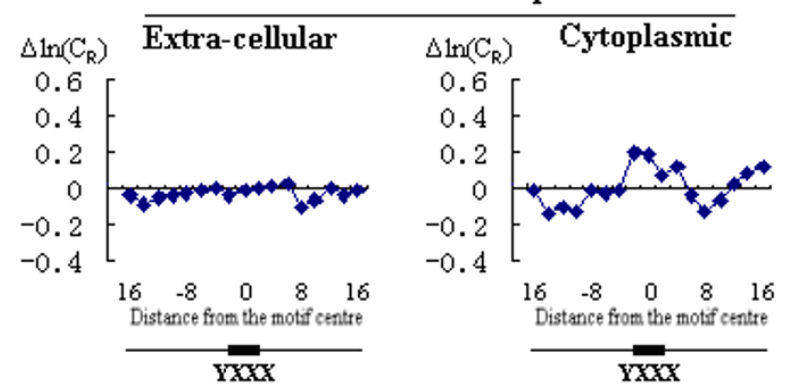

Receptor kinase/phosphatase

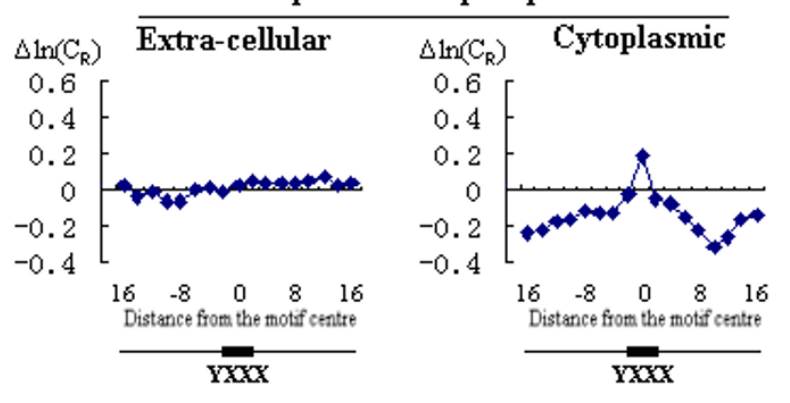

Adhesion molecule

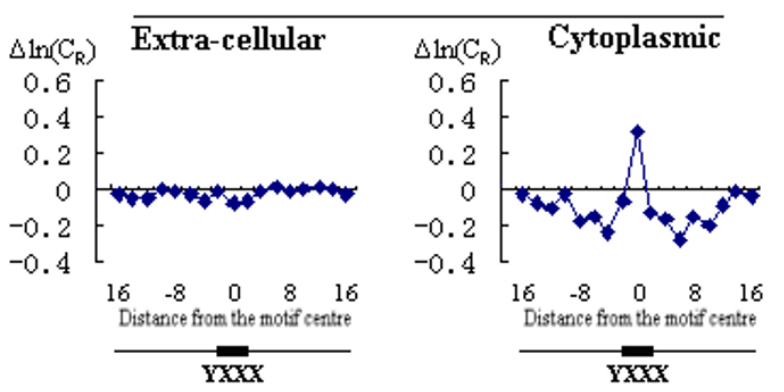

Figure 5

SH2-recognized Tyr-SLiMs in membrane proteins are conserved in the cytoplasmic but not the extra-cellular region. (A) The conservation signal in $\mathrm{SH} 2-$ recognized Tyr-SLiMs is stronger in the cytoplasmic region than in the extra-cellular region in both Type I and II membrane proteins, especially in Type I membrane proteins from specific functional classes (including cytokine/immune receptors, receptor kinases/phosphatases, cell surface receptors, and adhesion molecules). (B) Examples of conservation profiles that compare extra-cellular regions to cytoplasmic regions. 
A

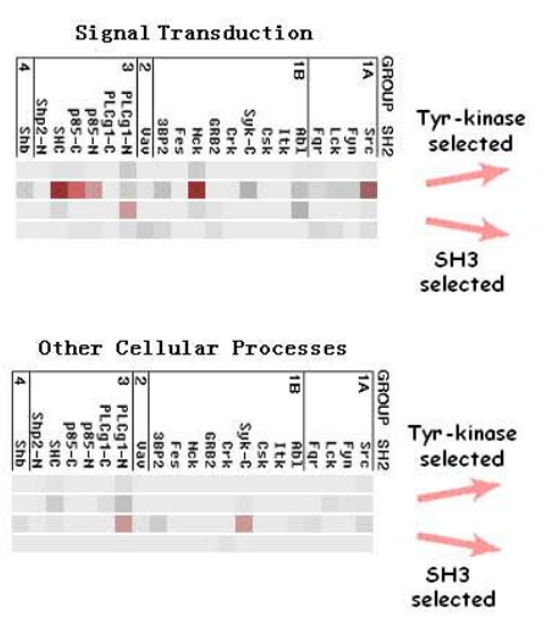

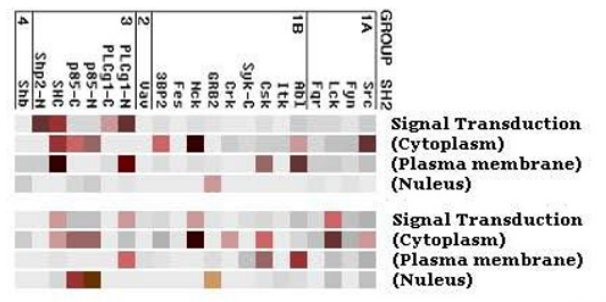
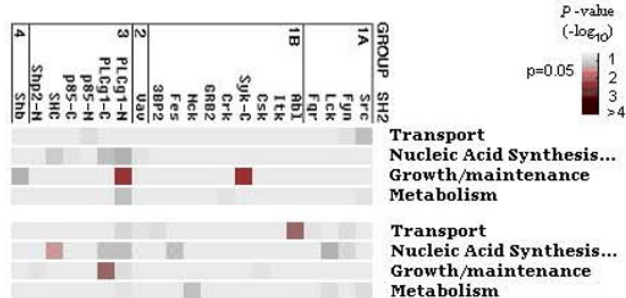

C
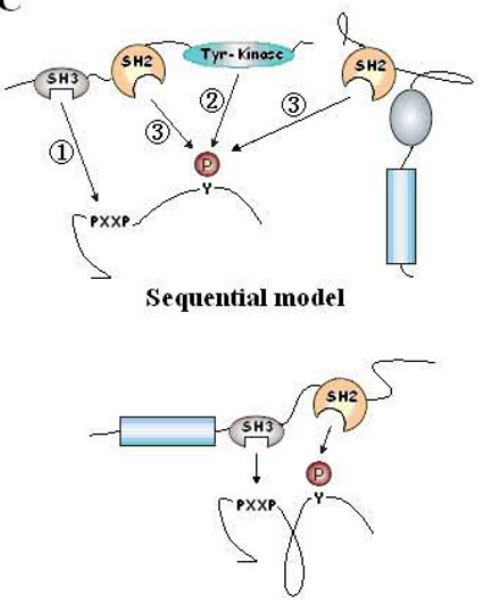

Cooperative model

B
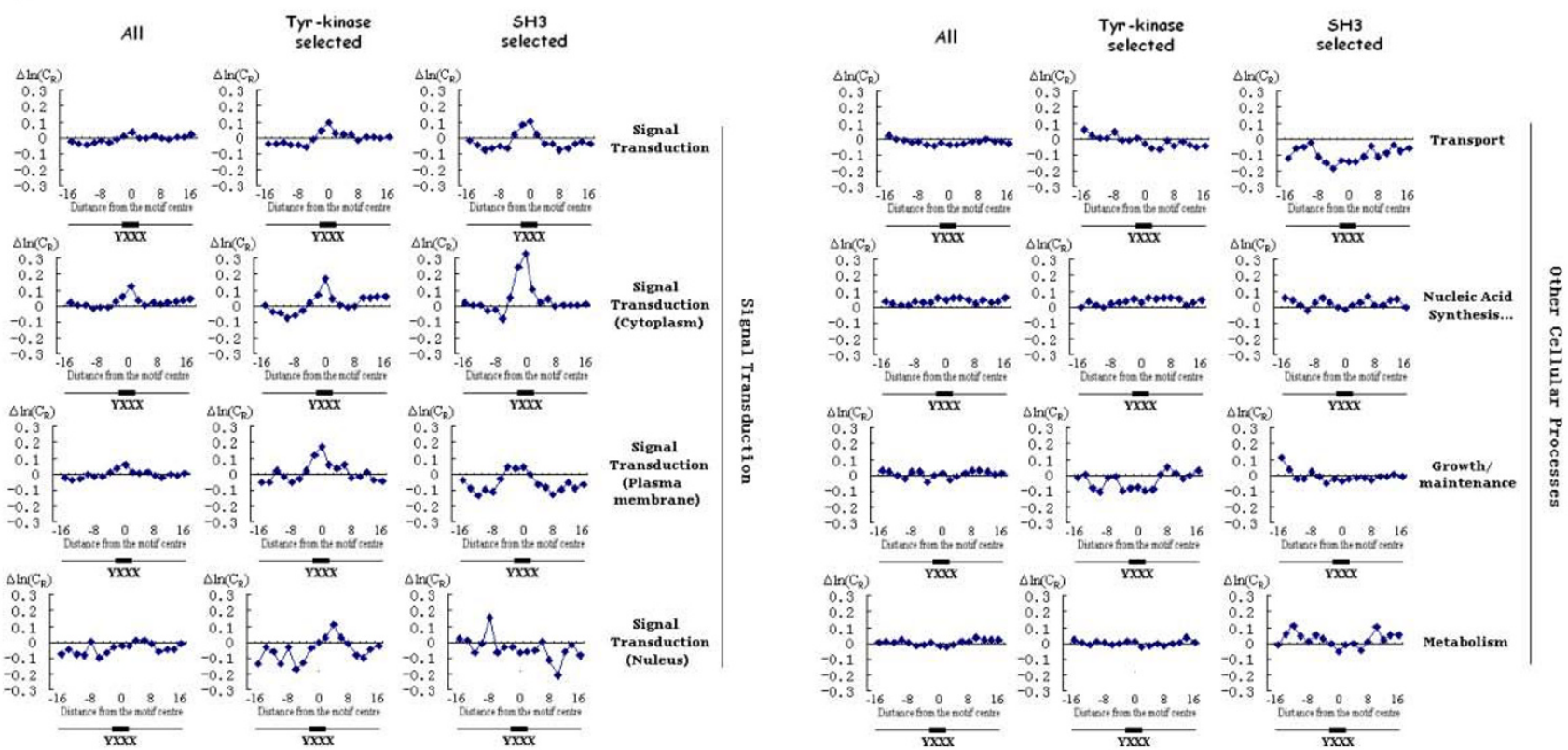

Figure 6

Conservation analysis of Tyr-SLiMs after tyrosine kinase and SH3 domain selection. (A) Comparison of relative conservation of SH2-recognized Tyr-SLiMs before and after Tyr-kinase and SH3 domain selections. (B) Conservation profile before and after Tyr-kinase and SH3 domain selections. (C) Schematic illustrations of the sequential model and the cooperative model to describe the coupling between $\mathrm{SH} 2$ and $\mathrm{SH} 3$ domains.

motif and the PXXP motif, respectively, to increase the strength of the interaction (Figure 6C, lower panel). Both of these models may explain the coupling between $\mathrm{SH} 2$ and $\mathrm{SH} 3$ domains. Early in tyrosine phosphorylationmediated signal transduction, most tyrosine residues are not phosphorylated, so the sequential model may prevail. However, after more tyrosine residues in signaling proteins become phosphorylated, the cooperative model may become increasingly relevant.

\section{Discussion}

Protein-protein interactions mediated by SLiMs have a widespread influence on cellular functions $[12,13]$. In this study, we examined these interactions by combining scoring matrixes derived from peptide library and conservation analysis. We detected signals of evolutionary conservation in SLiMs in proteins from functional classes that are known to participate in the signal transduction of a specific protein domain. Further, our analysis of membrane proteins indicated that only the cytoplasmic side is 
involved in SH2 signaling in both Type I and II membrane proteins. Our results also suggest that tyrosine kinase and SH3 domains are coupled with SH2 domain signaling in signal transduction proteins.

It was recently reported that several bacterially secreted cytotoxins contain multiple repeated Tyr-SLiMs with high affinity for both tyrosine kinases and SH2 domains [1417]. Many of these cytotoxins are phosphorylated upon entry into host cells and bind to a variety of SH2 proteins. For example, the CagA protein secreted by Helicobacter pylori can be phosphorylated by Src and associates with Shp2 [18] and Csk [18] SH2 domains, which is essential for cellular changes induced by the bacteria. The strong cellular response initiated by these $\mathrm{SH} 2$ binding TyrSLiMs further supports our assumption that SLiMs are under continuous evolutionary selection to preserve functional sites and eliminate harmful mutations. Recent work on the negative selection of $\mathrm{SH} 3$ domain-recognized sequences [19] also suggests that SLiMs may undergo strong evolutionary selection.

While most protein functional classes with strong conservation signal are known to be involved in the signaling of respective domains, there are a few exceptions, which may represent undiscovered but functional binding sites. For example, Although less than 3\% structural and cytoskeletal proteins have been recorded to bind to $\mathrm{SH} 2$ proteins, their Tyr-SLiMs selected by SH2 domains had significantly increased $C_{R}$ scores. It has been reported that alpha-Tubulin, a cytoskelatal protein, binds to the Fyn SH2 domain [20], and that the intermediate filaments of the cytokeratin type are reported to undergo tyrosine phosphorylation [21]. In the latter case, further evaluation is necessary to determine whether the phosphorylation leads to SH2 binding.

Another interesting observation is that DNA binding proteins also have conservation signal in their potential SH2 binding sites. Although tyrosine phosphorylation is generally believed to be less common in the nucleus, more and more evidences for the tyrosine phosphorylation of DNA binding proteins are reported as in the case of KRC DNA binding protein [22], estrogen receptor [23], TFII-I [24] and more examples provided in [25]. Since many SH2-containing proteins were reported to enter nucleus such as Fes [26], SHC [27], Nck [28] and Vav [29]. SH2 domains may mediate functional interactions with DNA binding proteins. Similar to SH2 domain, we observed that DNA binding proteins also have conservation signal in potential PDZ binding sites. Although most reported interactions mediated by PDZ domains are restricted to membrane proteins, proteins that contain PDZ domain (for example, LIM-kinase 1 [30] and Par3 [31]) were reported to enter nucleus suggesting they may mediate protein-protein interactions in the nucleus. Whether these observations represent a new trend of research is worth investigation.

Although our results from conservation analysis correlated well with biochemical data in general, our method is still prone to error. First, our motif prediction is based on in vitro peptide scanning techniques, which may be biased due to differences between in vitro and in vivo conditions. Second, we assumed that each position of the SLiM contributed equally to binding, and only SLiMs that were conserved at each position were assumed to be conserved. To improve this method in the future, different weights could be assigned to each position, and amino acid similarity could be considered. Finally, evolutionary conservation can only provide indirect clues regarding function. For example, some SLiMs may only be important for a few species, and these would not have been detected in our analysis.

Our results indicate that the conservation pattern of SLiMs recognized by $\mathrm{SH} 2, \mathrm{SH} 3, \mathrm{PDZ}$, and $\mathrm{S} / \mathrm{T}$ kinase domains highly correlates with the function of these domains. As motifs recognized by other domains are better defined, conservation analysis will be able to provide valuable clues as to their functional roles, as well as possible preferences for their sub-cellular localization or for their coupling with other domains and even structural implications. For example, in a recently published paper [32], the authors show that SLiMs are more likely to be conserved in disordered protein regions. Recently, peptide array based technology has been developed and is becoming increasingly available $[33,34]$. New technologies are expected to make motif discoveries easier and potentially more accurate. Currently, many of the motifs discovered are only defined as regular expressions, which usually provide less information than those motifs defined from the result of peptide library screening. Nevertheless, it should be possible to retrieve useful information from those less well-defined motifs using more sophisticated algorithms in the future.

\section{Conclusion}

This study systematically studied the evolutionary conservation of SLiMs recognized by $\mathrm{SH} 2, \mathrm{SH} 3, \mathrm{PDZ}$ and $\mathrm{S} / \mathrm{T}$ Kinase domains which reflected the interactome of these domains. Specifically, SLiMs within certain protein functional groups that are frequently involved in the interaction with that domain are significantly more conserved than those SLiMs within other groups. Study of manually extracted SH2 interaction sites in 11 most studied receptor tyrosine kinases provided experimental evidence that TyrSLiMs reported to interact with $\mathrm{SH} 2$ are significantly more conserved than those that do not. Furthermore, by analysis of SLiMs in membrane proteins and under selection of two different domains, we show that this conservation analysis can also provide useful information about the 
sub-cellular localization of the interaction and domain coupling.

\section{Methods \\ Selection and Classification of Human Protein Sequence Data}

We selected 7,248 human proteins for our protein functional classification analysis and 8,682 proteins for our cellular process classification analysis, using the following criteria: (1) The protein had SwissProt annotated sequence; (2) The protein had a molecular function or cellular process annotated by the Human protein reference database (Hprd) [35]; (3) The molecular function or cellular process of the protein was within 34 well-represented functional classes of proteins in Hprd.

Human protein sequence data are from the SwissProt database, downloaded from ftp://ftp.ncbi.nih.gov in November 2005. Protein-protein interactions, and classifications for protein molecular functions, biological processes and sub-cellular localizations are from the Hprd dataset [35]. This is a non-redundant manually-curated protein database, and data was downloaded in November 2005 from http://www.hprd.org. Phosphorylated sites were obtained from the Phospho.ELM database [36] provided by Francesca Diella in December 2005. We excluded several sequence regions unlikely to contain SLiMs (globular domains, coiled-coils, collagen regions and signal peptides, as annotated in SwissProt), because no more than $15 \%$ of known SLiMs $[12,37,38]$ occur in these regions.

\section{Selection of Homologous Proteins}

Using human protein sequences selected as described above, we did pair-wise local alignments generated by BLAST [39] against 12 higher eukaryotic species (Canis familiaris, Bos taurus, Mus musculus, Rattus norvegicus, Gallus gallus, Xenopus tropicalis, Tetraodon nigroviridis, Danio rerio, Strongylocentrotus purpuratus, Drosophila melanogaster, Apis mellifera, and Caenorhabditis elegans) to obtain homologous sequences for the respective human proteins. Species were selected according to their unique evolutionary positions (four mammals, four non-mammal vertebrates and four invertebrates) and sequence availability in the RefSeq database [40]. Sequence data for all non-human species were from the RefSeq database downloaded from ftp://ftp.ncbi.nih.gov in June 2006 except Tetraodon nigroviridis, which was from the NCBI Entrez non-redundant protein sequence database downloaded from ftp://ftp.ncbi.nih.gov in June 2006. We applied two cutoff levels to avoid inclusion of insignificant hits: a score cutoff of 50 bits, and an overlap cutoff of $50 \%$, as applied in Inparanoid [41]. If more than one homologous sequence was obtained from a single species, the one with the lowest E-value was selected. Unlike Inparanoid [41] or
COG (Cluster of Orthologous Groups) [42], which consider all species as equal entries, we compared sequences of all other species to those of human, because most biochemical data we used including protein interaction data and protein classification data were from human. Therefore, we only considered the best hit from non-human species as homologous to the human query protein, but not necessarily mutually best matches between human and non-human species or non-human species themselves. We have not removed low complexity regions because SLiMs frequently occur within them.

\section{Calculation of the Conservation Score of SLiMs}

SLiM occurrences were defined based on invariant features for each domain as listed in Table 1 (except ThrSLiMs were not included in the analysis for Ser/Thr kinases domains because only peptide library mapped motifs for Ser-SLiMs were available). All occurrences in the proteins that matched these invariant features were included in the analysis. For example, all sequences with the pattern YXXX were selected. For a particular protein sequence, we assumed that the sequence identity rate between a reference species (human in this study) and a species $\mathrm{i}$ is $\mathrm{p}_{(i)}$ (equal to the number of identical sites divided by the total number of sites aligned. In cases where gaps occur in the alignment sequence of species i, the number of gaps was subtracted from the number of sites aligned as the final alignment length), and that the SLiM under study is $\mathrm{n}$ amino acids in length (in cases where the SLiM is at the terminus of a protein and is only partially available, the available length was considered). If the SLiM is under the same evolutionary selectivity as the full-length protein, then the probability that the SLiM is conserved between the two species should be:

$$
\mathrm{P}_{1}(\mathrm{i})=\mathrm{p}(\mathrm{i})^{\mathrm{n}}
$$

The probability that the SLiM is unconserved should be:

$$
P_{2}(i)=1-P_{1}(i)=1-p(i)^{n}
$$

The SLiM is considered unconserved if any gap occurs within its sequence alignments.

Here we define Relative Conservation $\left(C_{R}\right)$ between human and the $\mathrm{i}^{\text {th }}$ species as:

a. if the SLiM is conserved:

$$
\mathrm{C}_{\mathrm{R}}(\mathrm{i})=1 / \mathrm{P}_{1}(\mathrm{i})=1 / \mathrm{p}(\mathrm{i})^{\mathrm{n}} ;
$$

b. if the SLiM is unconserved:

$$
\mathrm{C}_{\mathrm{R}}(\mathrm{i})=\mathrm{P}_{2}(\mathrm{i})=1-\mathrm{p}(\mathrm{i})^{\mathrm{n}} ;
$$


If $\mathrm{C}_{\mathrm{R}}(\mathrm{i})$ from $\mathrm{k}$ different species are $\left[\mathrm{C}_{\mathrm{R}}(1), \mathrm{C}_{\mathrm{R}}(2)\right.$, $\left.C_{R}(3), \ldots, C_{R}(k)\right]$, then $C_{R}$ of the SLiM among different species is calculated as:

$$
C_{R}=\sqrt[k]{\prod_{i=1}^{k} C_{R}(i)}
$$

A $C_{R}$ score greater than 1 indicates the SLiM is $C_{R}$ times more conserved than the average level of the protein. A score smaller than 1 indicates $1 / C_{R}$ times greater variability between species. Note that the number $k$ may be different for different SLiMs according to the pair-wise Blast results.

This method may not be suitable for SLiMs longer than 10 amino acids, since it assumes that most residues in the SLiM could influence the interaction. This may not be the case in longer sequences where only a small subset of the residues is critical to binding. This method was first developed in our lab and has demonstrated its effectiveness in another research[32] where SLiMs were found to be more conserved in disordered protein regions.

\section{Definition of Domain Selectivity}

For a putative SLiM, the selectivity value for domains were calculated as the product of enrichment values from peptide library experiments $[43,44]$. For example, to calculate the Src SH2 selectivity value of the SLiM YENF, we found the enrichment values for $\mathrm{E}(\mathrm{Y}+1)$ and $\mathrm{N}(\mathrm{Y}+2)$ for Src SH2 (Table 3) are 2.5 and 2.4, respectively. No enrichment value for $\mathrm{F}(\mathrm{Y}+3$ ) was found (thus $\mathrm{Y}+3$ does not contribute to the final value) and the selectivity value is the product of the two enrichment values $(2.5 \times 2.4=6.0)$. The enrichment values for $\mathrm{SH} 3$ domain recognized motifs were assigned based on amino acid sequence of peptides expressed by SH3-binding phage clones [45].

Please see Additional File 1 for more methods.

\section{Competing interests}

The authors declare that they have no competing interests.

\section{Authors' contributions}

SR was involved in design and planning of the experiments, has done the computational analysis and drafted the manuscript. GY, YH and YW were involved in carrying

Table 3: Enrichment values for the Src SH2 domain

\begin{tabular}{lcc}
\hline $\mathbf{P Y + 1}$ & $\mathbf{p Y + 2}$ & $\mathbf{p Y + 3}$ \\
\hline $\mathrm{E}(2.5)$ & $\mathrm{E}(2.6)$ & $\mathrm{I}(3.6)$ \\
$\mathrm{D}(1.7)$ & $\mathrm{N}(2.4)$ & $\mathrm{M}(2.5)$ \\
$\mathrm{T}(\mathrm{I} .7)$ & $\mathrm{Y}(2.0)$ & $\mathrm{L}(2.3)$
\end{tabular}

out experiments and computational analysis. YL was involved in planning of the experiments. ZC was involved in design and planning of the experiments, drafted the manuscript and headed the project. All authors have read and approved the final manuscript.

\section{Additional material}

\section{Additional file 1}

Additional methods and results. This additional file presents additional methods and results related to this article.

Click here for file

[http://www.biomedcentral.com/content/supplementary/14712164-9-452-S1.pdf]

\section{Acknowledgements}

We thank Wenchao Zhou and Uros Midic for comments on this manuscript. We are grateful to Dr. Michael B. Yaffe, Dr. Tieliu Shi, Dr. Longhou Fang, Li Zhuo and Dan Du for useful criticism and discussions. This work was supported by the grants from National Natural Science Foundation of China (No. 30730055 and No. 30623002) and from the National Key Scientific Program of China (No. 2007CB914504) and also from the National High Technology Research and Development Program of China (No. 2006AA02A308).

\section{References}

I. Songyang Z, Shoelson SE, Chaudhuri M, Gish G, Pawson T, Haser WG, King F, Roberts T, Ratnofsky S, Lechleider RJ, et al: SH2 domains recognize specific phosphopeptide sequences. Cell 1993, 72(5):767-778.

2. Songyang Z, Shoelson SE, McGlade J, Olivier P, Pawson T, Bustelo XR, Barbacid M, Sabe H, Hanafusa $H$, Yi T, et al.: Specific motifs recognized by the SH2 domains of Csk, 3BP2, fps/fes, GRB-2, HCP, SHC, Syk, and Vav. Mol Cell Biol I994, I 4(4):2777-2785.

3. Yaffe MB, Leparc GG, Lai J, Obata T, Volinia S, Cantley LC: A motifbased profile scanning approach for genome-wide prediction of signaling pathways. Nat Biotechnol 200I, 19(4):348-353.

4. Obenauer JC, Cantley LC, Yaffe MB: Scansite 2.0: Proteome-wide prediction of cell signaling interactions using short sequence motifs. Nucleic Acids Res 2003, 3 I (13):3635-364I.

5. Balla S, Thapar V, Verma S, Luong T, Faghri T, Huang CH, Rajasekaran S, del Campo JJ, Shinn JH, Mohler WA, et al: Minimotif Miner: a tool for investigating protein function. Nat Methods 2006, 3(3): 175-177.

6. Gutman R, Berezin C, Wollman R, Rosenberg Y, Ben-Tal N: QuasiMotiFinder: protein annotation by searching for evolutionarily conserved motif-like patterns. Nucleic Acids Res 2005:W255-26I.

7. Dinkel H, Sticht $\mathrm{H}$ : A computational strategy for the prediction of functional linear peptide motifs in proteins. Bioinformatics 2007, 23(24):3297-3303.

8. Chica C, Labarga A, Gould CM, Lopez R, Gibson T): A tree-based conservation scoring method for short linear motifs in multiple alignments of protein sequences. BMC Bioinformatics 2008, 9:229.

9. Kashiwada M, Giallourakis CC, Pan PY, Rothman PB: Immunoreceptor tyrosine-based inhibitory motif of the IL-4 receptor associates with $\mathrm{SH} 2$-containing phosphatases and regulates IL-4-induced proliferation. J Immunol 200 I, I67(II):6382-6387.

10. Pellicena P, Miller WT: Processive phosphorylation of p I30Cas by Src depends on SH3-polyproline interactions. J Biol Chem 2001, 276(30):28190-28196.

II. Nakamoto T, Sakai R, Ozawa K, Yazaki Y, Hirai H: Direct binding of C-terminal region of p $130 \mathrm{Cas}$ to $\mathrm{SH} 2$ and $\mathrm{SH} 3$ domains of Src kinase. J Biol Chem 1996, 27 I ( 1 5):8959-8965. 
12. Neduva V, Linding R, Su-Angrand I, Stark A, de Masi F, Gibson T], Lewis J, Serrano L, Russell RB: Systematic discovery of new recognition peptides mediating protein interaction networks. PLoS Biol 2005, 3( I 2):e405.

13. Pawson T, Scott JD: Signaling through scaffold, anchoring, and adaptor proteins. Science 1997, 278(5346):2075-2080.

14. Campellone KG, Leong JM: Nck-independent actin assembly is mediated by two phosphorylated tyrosines within enteropathogenic Escherichia coli Tir. Mol Microbiol 2005, 56(2):416-432.

15. Backert S, Selbach M: Tyrosine-phosphorylated bacterial effector proteins: the enemies within. Trends Microbiol 2005, 13(10):476-484.

16. Clifton DR, Fields KA, Grieshaber SS, Dooley CA, Fischer ER, Mead DJ, Carabeo RA, Hackstadt T: A chlamydial type III translocated protein is tyrosine-phosphorylated at the site of entry and associated with recruitment of actin. Proc Natl Acad Sci USA 2004, I0I(27): I0166-1017I.

17. Schulein R, Guye P, Rhomberg TA, Schmid MC, Schroder G, Vergunst AC, Carena I, Dehio C: A bipartite signal mediates the transfer of type IV secretion substrates of Bartonella henselae into human cells. Proc Natl Acad Sci USA 2005, 102(3):856-86I.

18. Tsutsumi R, Higashi H, Higuchi M, Okada M, Hatakeyama M: Attenuation of Helicobacter pylori CagA $\times$ SHP-2 signaling by interaction between CagA and C-terminal Src kinase. J Biol Chem 2003, 278(6):3664-3670.

19. Zarrinpar A, Park SH, Lim WA: Optimization of specificity in a cellular protein interaction network by negative selection. Nature 2003, 426(6967):676-680.

20. Klein C, Kramer EM, Cardine AM, Schraven B, Brandt R, Trotter J: Process outgrowth of oligodendrocytes is promoted by interaction of fyn kinase with the cytoskeletal protein tau. J Neurosci 2002, 22(3):698-707.

21. Strnad P, Windoffer R, Leube RE: Induction of rapid and reversible cytokeratin filament network remodeling by inhibition of tyrosine phosphatases. J Cell Sci 2002, I I 5(Pt 2I):4I33-4I48.

22. Bachmeyer C, Mak CH, Yu CY, Wu LC: Regulation by phosphorylation of the zinc finger protein KRC that binds the kappaB motif and $V(D) J$ recombination signal sequences. Nucleic Acids Res 1999, 27(2):643-648.

23. Arnold SF, Obourn JD, Jaffe H, Notides AC: Phosphorylation of the human estrogen receptor on tyrosine 537 in vivo and by src family tyrosine kinases in vitro. Mol Endocrinol 1995, 9(I):24-33.

24. Novina CD, Kumar S, Bajpai U, Cheriyath $V$, Zhang K, Pillai S, Wortis $\mathrm{HH}$, Roy AL: Regulation of nuclear localization and transcriptional activity of TFII-I by Bruton's tyrosine kinase. Mol Cell Biol 1999, 19(7):5014-5024

25. Cans C, Mangano R, Barila D, Neubauer G, Superti-Furga G: Nuclear tyrosine phosphorylation: the beginning of a map. Biochem Pharmacol 2000, 60(8): I203-1215.

26. Yates KE, Lynch MR, Wong SG, Slamon DJ, Gasson JC: Human cFES is a nuclear tyrosine kinase. Oncogene 1995, I0(6): $1239-1242$

27. Huang H, Li L, Wu C, Schibli D, Colwill K, Ma S, Li C, Roy P, Ho K, Songyang Z, et al.: Defining the specificity space of the human SRC homology 2 domain. Mol Cell Proteomics 2008, 7(4):768-784.

28. Kremer BE, Adang LA, Macara IG: Septins regulate actin organization and cell-cycle arrest through nuclear accumulation of NCK mediated by SOCS7. Cell 2007, 130(5):837-850.

29. Romero F, Fischer S: Structure and function of vav. Cell Signal 1996, 8(8):545-553.

30. Yokoo T, Toyoshima H, Miura M, Wang Y, lida KT, Suzuki H, Sone H, Shimano $\mathrm{H}$, Gotoda $\mathrm{T}$, Nishimori $\mathrm{S}$, et al:: p57Kip2 regulates actin dynamics by binding and translocating LIM-kinase I to the nucleus. J Biol Chem 2003, 278(52):52919-52923.

31. Fang L, Wang Y, Du D, Yang G, Tak Kwok T, Kai Kong S, Chen B, Chen DJ, Chen Z: Cell polarity protein Par3 complexes with DNA-PK via Ku70 and regulates DNA double-strand break repair. Cell Res 2007, I7(2): I00-II6.

32. Ren S, Uversky V, Chen Z, Dunker AK, Obradovic Z: Short Linear Motifs recognized by $\mathrm{SH} 2, \mathrm{SH} 3$ and Ser/Thr Kinase domains are conserved in disordered protein regions. BMC Genomics 2008, 9 Suppl 2:S26.

33. Peri S, Navarro JD, Amanchy R, Kristiansen TZ, Jonnalagadda CK, Surendranath V, Niranjan V, Muthusamy B, Gandhi TK, Gronborg M, et al.: Development of human protein reference database as an initial platform for approaching systems biology in humans. Genome Res 2003, I3(10):2363-237I.

34. Li L, Wu C, Huang H, Zhang K, Gan J, Li SS: Prediction of phosphotyrosine signaling networks using a scoring matrix-assisted ligand identification approach. Nucleic Acids Res 2008.

35. Peri S, Navarro JD, Kristiansen TZ, Amanchy R, Surendranath $V$, Muthusamy B, Gandhi TK, Chandrika KN, Deshpande N, Suresh S, et al.: Human protein reference database as a discovery resource for proteomics. Nucleic Acids Res 2004:D497-50I.

36. Diella F, Cameron S, Gemund C, Linding R, Via A, Kuster B, SicheritzPonten T, Blom N, Gibson T]: Phospho.ELM: a database of experimentally verified phosphorylation sites in eukaryotic proteins. BMC Bioinformatics 2004, 5:79.

37. Puntervoll $P$, Linding R, Gemund $C$, Chabanis-Davidson $S$, Mattingsdal M, Cameron S, Martin DM, Ausiello G, Brannetti B, Costantini A, et al.: ELM server: A new resource for investigating short functional sites in modular eukaryotic proteins. Nucleic Acids Res 2003, 3 I ( I 3):3625-3630.

38. lakoucheva LM, Radivojac P, Brown CJ, O'Connor TR, Sikes JG, Obradovic Z, Dunker AK: The importance of intrinsic disorder for protein phosphorylation. Nucleic Acids Res 2004, 32(3): 1037-1049.

39. Altschul SF, Madden TL, Schaffer AA, Zhang J, Zhang Z, Miller W, Lipman DJ: Gapped BLAST and PSI-BLAST: a new generation of protein database search programs. Nucleic Acids Res 1997, 25(17):3389-3402.

40. Pruitt KD, Tatusova T, Maglott DR: NCBI Reference Sequence (RefSeq): a curated non-redundant sequence database of genomes, transcripts and proteins. Nucleic Acids Res 2005:D50I-504

4I. Remm M, Storm CE, Sonnhammer EL: Automatic clustering of orthologs and in-paralogs from pairwise species comparisons. I Mol Biol 2001 , 3 I 4(5): 104I-1052.

42. Tatusov RL, Fedorova ND, Jackson JD, Jacobs AR, Kiryutin B, Koonin EV, Krylov DM, Mazumder R, Mekhedov SL, Nikolskaya AN, et al: The COG database: an updated version includes eukaryotes. BMC Bioinformatics 2003, 4:41.

43. Manke IA, Nguyen A, Lim D, Stewart MQ, Elia AE, Yaffe MB: MAP. KAP kinase-2 is a cell cycle checkpoint kinase that regulates the $G 2 / M$ transition and $S$ phase progression in response to UV irradiation. Mol Cell 2005, I 7(I):37-48.

44. Songyang Z, Cantley LC: The use of peptide library for the determination of kinase peptide substrates. Methods Mol Biol 1998, 87:87-98.

45. Sparks AB, Rider JE, Hoffman NG, Fowlkes DM, Quillam LA, Kay BK: Distinct ligand preferences of Src homology 3 domains from Src, Yes, Abl, Cortactin, p53bp2, PLCgamma, Crk, and Grb2. Proc Natl Acad Sci USA 1996, 93(4): I 540 - I544.

Publish with Bio Med Central and every scientist can read your work free of charge

"BioMed Central will be the most significant development for disseminating the results of biomedical research in our lifetime. "

Sir Paul Nurse, Cancer Research UK

Your research papers will be:

- available free of charge to the entire biomedical community

- peer reviewed and published immediately upon acceptance

- cited in PubMed and archived on PubMed Central

- yours - you keep the copyright
BioMedcentral 